\title{
EL SENTIMIENTO DE FUNDAMENTALIDAD EN ZUBIRI
}

\author{
ESTEBAN VARGAS, EUGENIA COLOMER \\ Pontificia Universidad Católica de Valparaíso
}

\begin{abstract}
RESUMEN: El escrito intenta desarrollar el papel que jugaría el sentimiento en la fundamentalidad en Zubiri, lo que se expresa en diversas actitudes ante el problema de Dios (teísmo, ateísmo, etc.). Proponemos que el sentimiento permite, por una parte, dirigir la creación de diversas posibilidades de realización a través de juicios de valor. Dirige, en cierto modo, la búsqueda de estas diversas actitudes. Por otra parte, permite la «probación» o experiencia de los esbozos de esas actitudes. Por ejemplo, la experiencia negativa de una determinada concepción de Dios es condicionada no sólo por aspectos intelectivos sino de sentimiento. Damos algunos ejemplos de ello sacados de la vida de algunos filósofos.
\end{abstract}

PALABRAS CLAVES: sentimiento; intelección; volición; fundamentalidad; Zubiri.

\section{The feeling of fundamentality in Zubiri}

ABSTRACT: The paper tries to develop the role that the feeling of fundamentality plays in Zubiri. This feeling is expressed in diverse attitudes towards the problem of God (theism, atheism, etc.). We propose that the feeling allows, in part, to direct the creation of diverse possibilities of realization through value judgments. It directs, to a certain extent, the search for these diverse attitudes. On the other hand, it allows for the experience of the sketches of these attitudes. For instance, the negative experience of a determined conception of God is conditioned not only by intellective aspects, but also sentimental ones. We give some examples of this out of some philosophers' lives.

KEY WORDS: feeling; intellection; volition; fundamentality; Zubiri.

\section{INTRODUCCIÓN}

Estudiar el tema del sentimiento de fundamentalidad en Zubiri es de interés por tres razones principales. En primer lugar, porque es relevante meditar qué papel juega el sentimiento como momento de la actitud radical frente a la realidad. En segundo lugar, y ya entrando en la obra de Zubiri, por la relevancia que tiene el tema de la fundamentalidad en su filosofía. Este tema fue atendido tempranamente por el autor, conservando una cierta uniformidad en su tratamiento a lo largo de su obra, y que trae, además, una llamativa novedad dentro de la tradición filosófica, tal como lo señala Diego Gracia ${ }^{1}$. Y, en tercer lugar, y pese a la importancia del tema, porque Zubiri no trató explícitamente el papel del sentimiento en el problema de la fundamentalidad de lo real (también llamado por él problema de Dios o teologal). Por ejemplo, no indicó qué papel juega el sentimiento en las actitudes ante el problema de la fundamentalidad como el teísmo, ateísmo, etc. El problema de la fundamentalidad fue estudiado sólo desde la intelección y la voluntad, pero no explícitamente desde el sentimiento.

1 Cf. Gracia, D., (2017). El poder de lo real. Leyendo a Zubiri, Madrid: Triacastela, p. 380. 
Nuestra tarea es entonces postular el posible papel que jugaría el sentimiento en el problema de la fundamentalidad en y desde Zubiri.

Para poder tratar este último punto dividimos el escrito en tres partes. En primer lugar, decir brevemente qué es sentimiento. En segundo lugar, indicar qué es intelección y volición de fundamentalidad, temas que Zubiri sí trató un poco más. Finalmente, propondremos en qué podría consistir el sentimiento de fundamentalidad.

\section{Qué es Sentimiento}

Según Zubiri, en todo animal (incluyendo al hombre) se puede analizar su sentir desde su momento procesual en tres momentos: suscitación, modificación tónica y respuesta. En el caso del hombre, estos tres momentos nos presentan o actualizan la realidad de tres modos distintos: como intelección sentiente, sentimiento afectante y volición tendente. No son tres modos separados de aprehensión sino que están intrínsecamente articulados. Veamos esto muy brevemente, destacando principalmente el momento del sentimiento.

La intelección sentiente es el momento de mera actualización impresiva de lo real. Al momento de impresión Zubiri le llama «sentir» y al momento de realidad «inteligir». No son dos actos distintos sino un mismo acto atendido desde dos respectos. Equivale al momento de «suscitación» de todo sentir animal. La volición tendente, por su parte, atiende al momento de «respuesta» animal, que en el hombre es radicalmente una apropiación de lo real. Las cosas no sólo se actualizan como meramente reales, sino como algo que tiene que ser apropiado. Y ¿qué decir del sentimiento afectante?

Este cometido presenta algunas dificultades, porque el tema del sentimiento, no obstante su gran importancia en la obra de Zubiri, tiene un escaso y tardío tratamiento, manifestado principalmente en su curso «Reflexiones filosóficas sobre lo estético» (1975) y sus libros Inteligencia sentiente (1980-1983) y El hombre y Dios (1983) que quedó inconcluso. El tema, como el mismo autor consigna, debe atenderse desde dos momentos: «[...] el sentimiento como acto, y la realidad que en ese acto nos está presente»².

\section{a) El sentimiento como acto}

El sentimiento es, ante todo, un momento del proceso sentiente, a saber, el momento de «modificación tónica» del sentir animal. En esto difiere de los otros momentos del proceso sentiente como intelección y volición. Esto no significa que intelección, volición y sentimiento sean tres actos numéricamente independientes. Son, en rigor, tres momentos de un mismo acto de aprehensión.

2 ZUBIRI, X. (1992). Sobre el sentimiento y la volición (SSV). Madrid: Alianza, p. 336. Los libros de Zubiri, como ya es tradicional, se citan por sus siglas. 
Por otra parte, el sentimiento como acto nos da ese momento de ser algo aprehendido como «mío», tal como ocurre en la intelección y la volición. En el caso del sentimiento, quedamos «afectados» como en la ira, pena, etc. Esto lleva a creer, sobre todo en el sentimiento, que es un acto puramente «subjetivo». Pero esto ocurre por desatender al segundo momento del sentimiento, inseparable del anterior, «la realidad que en ese acto nos está presente». En el hombre todo sentimiento se actualiza en formalidad de realidad. Por ello, los sentimientos no son meras modificaciones tónicas animales. ¿Cómo se nos actualiza lo real en esta modificación tónica?

\section{b) La realidad actualizada en el sentimiento}

En el hombre, en nuestros sentimientos (ira, rabia, etc.), lo real se actualiza como un modo de estar "atemperado» a ella. En todos esos sentimientos no sólo sentimos diversos contenidos, sino que estamos acomodados o no en la realidad. No es que exista algo que empiece por presentarse como real, y que luego nos acomode o no, sino que en nuestros sentimientos lo real se actualiza «de suyo» como atemperante (cómodo o incómodo). El sentimiento es un modo de actualización de lo real, es «un modo de estar realmente en la realidad, o si se quiere, es sentirse realmente en la realidad $»^{3}$. Por ello, el sentimiento no es algo meramente subjetivo, sino que en él se actualiza la realidad, la que por ser «de suyo» es siempre abierta, es un «más» al contenido tonal de todo sentimiento. La realidad es "de suyo» tonal. A ese carácter tonal de la realidad Zubiri lo denomina temperie ${ }^{4}$. Es el momento de fruición, de degustación de lo real por el cual nos sentimos acomodados o no a ella. Finalmente, desde este respecto, hay que distinguir dos momentos de todo sentimiento. Por un lado, el sentimiento de las «cosas reales», considerando predominantemente sus contenidos o cualidades. En este sentido, los sentimientos son diversos. Pero hay otro momento, inseparable del anterior, que es el momento de «la» realidad, su momento transcendental. A este momento Zubiri le llamará «sentimiento estético» ${ }^{5}$. El sentimiento estético no hace referencia a un sentimiento más, entre otros, sino «es el momento "real" de todo sentimiento, es la fruición de lo real como real en todo sentimiento ${ }^{6}$. Es el goce de la actualidad de la realidad en tanto realidad, lo real como pulchrum.

Ahora bien, del mismo modo como ocurre en el despliegue de los modos de intelección en tres momentos (aprehensión primordial, logos y razón), Zubiri «parece» hacer lo mismo en el sentimiento, momentos que llama «en su realidad» (se actualiza lo real como hermoso o feo), «por ser reales» (sólo como

\footnotetext{
3 SSV, pp. 332-333.

4 Valentina Bulo recoge la acepción de tono de la realidad como tensión, carácter de la realidad que en la realidad humana se actualiza a modo de transtensidad. Cf. Bulo, V., Tonos de realidad. Pensar el sentimiento en la filosofía de Xavier Zubiri, Santiago: RIL editores, p. 71.

5 Cf. SSV, p. 346.

6 SSV, p. 349.
} 
bello) y «en cuanto realidad» (como pulchrum). Las dos primeras se refieren predominantemente a la actualidad de la cosa real, la tercera a su momento más inespecífico de realidad. Como Diego Gracia señala en una nota a pie de página en $\mathrm{SSV}^{7}$, la distinción de estos estratos recuerda la correspondencia con los modos de intelección — «en su realidad» (razón), «por ser reales» (logos) y «en cuanto realidad» (aprehensión primordial)_, aunque todavía de un modo impreciso, por ser un texto anterior a la trilogía de Inteligencia sentiente. Pero, sea ello o no verdad ${ }^{8}$, lo que aquí nos interesa destacar es que el sentimiento posee distintos estratos o modos de actualidad de lo real, cuestión sobre la que volveremos más abajo.

\section{INTELECCIÓN Y VOLICIÓN DE FUNDAMENTALIDAD}

Zubiri destaca en este tema dos modos de la actualidad de lo real: la realidad-objeto y la realidad-fundamento (fundamentalidad). Las estudia desde el momento de intelección y de volición. Veámoslas brevemente, destacando en especial su momento de fundamentalidad.

\section{a) Intelección de fundamentalidad}

El tema del fundamento, desde el punto de vista intelectivo, aparece desarrollado, por un lado, en la tercera parte de Inteligencia sentiente llamada Inteligencia y razón. Allí se nos dice que la razón nos «actualiza» lo real desde su fundamento: «Fundamento es todo aquello que determina desde sí mismo, pero en y por sí mismo, a lo percibido, de suerte que éste es una realización del fundamento en lo fundado mismo» ${ }^{9}$. Este fundamento es, para Zubiri, algo que, en cierta forma, nos es dado por la aprehensión misma de realidad como «búsqueda». Lo que no nos es dado es «cuál» sea el «contenido» del fundamento de lo real. A este momento fundante de la realidad es lo que Zubiri llama «realidadfundamento». Pero no hay fundamento sin algo fundado. Esa realidad fundada es lo que Zubiri llama «realidad-objeto». Realidad-objeto y realidad-fundamento son los dos modos en que se nos actualiza lo real en la razón ${ }^{10}$. Por otro lado, el tema del fundamento, en forma más amplia, no sólo intelectiva, es tratado

7 SSV, pp. 362-363.

8 Esto no es tan claro. Por ejemplo, Zubiri señala que «cada estrato se funda en el anterior» (SSV, p. 372), lo que no ocurre en Inteligencia sentiente, pues, por ejemplo, el logos no se funda en la razón, que sería su estrato anterior, según la lectura de Diego Gracia.

9 ZuBIRI, X. (1983). Inteligencia y razón (IRA). Madrid: Alianza, p. 161.

10 Diego Gracia distingue en este tema tres órdenes: talitativo (propio de la realidadobjeto), transcendental (aprehensión primordial) y transcendente (razón). El fundamento es transcendente en tanto busca más allá de la aprehensión. Por ello dice: «Las cosas son objetos; la realidad es trascendental, y el fundamento trascendente». Gracia, D., (2017). El poder de lo real. op. cit., p. 399. 
también en El hombre y Dios ${ }^{11}$. Allí se nos dice que el hombre hace su vida «con» las cosas reales en «la» realidad. «La» realidad como formalidad es «más» que sus contenidos, es decir, «domina» o tiene "poder» sobre ellos, aunque no es un «más» sin ellos. Este poder de lo real nos impele o liga a realizarnos: «Esta peculiar ligadura es justo religación» ${ }^{12}$. El hombre se encuentra religado al poder de lo real. Este poder al que el hombre está religado posee como fundamentalidad un carácter último, posibilitante e impelente. La realidad es «última» en tanto que el hombre hace su vida «en» ella. Es «posibilitante» en tanto hace su vida «desde» ella. Finalmente, es «impelente» en tanto hace su vida "por» ella. De los tres momentos, el carácter «último» es el que parece "predominar» más (sin anular los dos restantes) en el aspecto «intelectivo» de la fundamentalidad ${ }^{13}$. El hombre está impelido a buscar cuál es el "contenido fundamental» de lo real en tanto real, es decir, en qué consiste su «ultimidad». Fue el aspecto que más predominó en el pasado en las pruebas de la existencia de Dios.

\section{b) Volición de fundamentalidad}

Pero la religación, para Zubiri, no es sólo un aspecto intelectivo. Es un hecho integral de todo el hombre ${ }^{14}$. Y aquí el aspecto volitivo también juega un papel crucial en la fundamentalidad. Para aclarar esto, consideremos que, según los modos ya señalados de actualidad de lo real, hay en el fondo dos tipos de volición. Por una parte, frente a la realidad-objeto, en una volición puedo, si quiero, tomar una opción por sobre otras. Aquí hay una volición facultativa. Como dice Zubiri: «Yo podría, por ejemplo, facultativamente no ocuparme de astronomía ${ }^{15}$. Pero, por otra parte, al mismo tiempo que elijo tal o cuál opción frente a cada cosa estoy eligiendo para mí una «forma de realidad» por sobre otras. Y éste es el aspecto de volición radical: «Para los efectos de la volición radical, el término de ella no se presenta como objeto o cosa sino que su modo de estar presente es estar fundamentando nuestra realidad relativamente absoluta. No es una cosa que "está" presente sino que su modo de estarlo es "fundamentar" ${ }^{16}$. Es la volición de fundamentalidad. La fundamentalidad no

11 Para más detalles de todo lo que sigue sobre la intelección y volición de fundamentalidad puede verse VARGAS, E. (2015). «Filosofía y el problema de Dios» en Pensamiento, vol. 71, núm. 266, pp. 491-504.

12 Zubiri, X. (2012). El hombre y Dios. Nueva Edición (HD). Madrid: Alianza, p. 98.

13 Para Pintor-Ramos, los tres momentos de la fundamentalidad se relacionan con los tres momentos de la aprehensión de lo real: «La religación es la dominancia real sobre las notas que configuran la persona como realidad última (aspecto intelectivo), posibilitante (aspecto sentimental) e impelente (aspecto volitivo) y esa religación es la que actualiza la realidad como "fundamento"». Cf. Pintor-Ramos, A. (2015). «Desarrollo del concepto de religación en Zubiri» en Cuadernos Salmantinos de Filosofía, Vol. 42, p. 124. Nosotros seguiremos esta idea como esbozo de lo que podría ser el sentimiento de fundamentalidad.

14 Cf. HD, pp. 98-99.

15 HD, p. 434.

16 HD, pp. 109-110. 
es algo que esté meramente «ahí» (no es realidad-objeto), sino que su realidad consiste en «impelerme» inexorablemente a tomar una opción frente a ella. No se puede no optar en cada acción por una forma de realidad. No es una volición facultativa. Si, por ejemplo, se elige un día pasear, no sólo se elige eso, sino también se está optando por una «forma de realidad»: ser un caminante, y no un sedentario. Estamos «impelidos» a darnos siempre una forma de realidad. De allí la gravedad para Zubiri de todos los actos de la vida: son transcendentales. La fundamentalidad no es, entonces, sólo un problema racional sobre la ultimidad de lo real (por ejemplo, sobre el fundamento del mundo), uno más entre los muchos que el hombre pueda tener, sino que es un problema de «realización» personal que involucra, a una, su intelección y volición radical: es la voluntad de verdad real ${ }^{17}$. De los tres momentos de la fundamentalidad, el momento de «impelencia» para ser real parece ser el que más "predomina» o destaca en la volición radical. Estamos impelidos a optar en cada acción concreta por una forma de realidad.

De la unión de estos dos momentos (intelectivo y volitivo) se originan, para Zubiri, las diversas «actitudes» ante la fundamentalidad. Por ejemplo: teísmo, ateísmo, indiferentismo, agnosticismo, etc. No se trata de considerarlas, como es usual, en su aspecto puramente «intelectivo»: ellas no consisten meramente en afirmar, negar, suspender, ignorar, etc., la «creencia» en Dios como ser supremo. Los nombres que reciben estas actitudes suelen referirse sólo a este punto. Pero, para Zubiri, lo «radical» de estas actitudes es servir de «inspiración» para tomar postura intelectiva y volitiva no frente a la creencia de una realidad-objeto suprema, sino ante la fundamentalidad, lo cual es algo inexorable. Así nos dice Zubiri: «Este resultado [de búsqueda] no es unívoco, sino que la búsqueda puede estar inspirada en algo previo que llamaremos actitud. Cada dirección de búsqueda constituye una actitud ${ }^{18}$. Por eso, cada dirección de búsqueda (teísmo, ateísmo, etc.) es, para Zubiri, precisamente una "actitud», algo previo al «acto» de tomar postura racional y entrega ante la fundamentalidad.

Ahora bien, en estas actitudes se trata, pues, no sólo de buscar el contenido fundamental, sino también tomar necesariamente una postura volitiva (entrega) ante esa fundamentalidad en cada acción. En cada acción del hombre se juega una posible solución ante el problema de la fundamentalidad. Estos dos momentos (intelectivo y volitivo de fundamentalidad) pueden darse en diversos grados. Por ejemplo, hay personas que con un mínimo de conocimiento sobre la fundamentalidad pueden tener una gran entrega radical en cada acción o, al revés, puede haber un gran conocimiento con un mínimo de entrega. Todo ello hace que Zubiri haya repensado las clásicas posturas «intelectivas» ante Dios

17 Así Zubiri nos dice: «En cada uno de sus actos el hombre está ejecutando una volición de verdad real. En ella tiene que adoptar una forma de realidad. Esta forma es, pues, optativa. Por tanto, la voluntad de verdad real se plasma en búsqueda. ¿Búsqueda de qué? Búsqueda de cómo se articulan las cosas reales en "la" realidad para poder optar por una forma de realidad» (HD, p. 112).

18 HD, p. 123. 
como diversas «actitudes» radicales ante el problema de la fundamentalidad desde un esquema bipartito de intelección y volición. Pero si la religación es un hecho integral, ¿jugará el sentimiento un papel en la fundamentalidad? ¿Atenderá predominantemente al momento de «posibilidad» de la fundamentalidad? Es lo que intentaremos esbozar ahora.

\section{Sentimiento DE FUndamentalidad}

El hombre, como decíamos, está fundado «en» (ultimidad), «desde» (posibilitancia) y "por» (impelencia) la realidad. La realidad es el apoyo para ser persona, es «fundamento de la persona ${ }^{19}$. En este escrito queremos «esbozar» el modo de actualización propio del poder de lo real en el sentimiento desde dos momentos. Por una parte, su posible papel «posibilitante» en el despliegue «hacia» la realidad-fundamento que realizan las diversas actitudes. Por otra parte, su posible papel en la "probación» o experiencia de la propia realización personal. Estos dos aspectos no agotan todo lo que pueda decirse sobre el sentimiento de fundamentalidad, pero sirven para esbozar su papel en la realización del hombre.

\section{a) El carácter posibilitante del sentimiento de fundamentalidad}

Decíamos que el hombre para «realizarse» se ve impelido a optar por una forma de realidad entre otras en cada acción de su vida. Tal realización es problemática porque el hombre en cada acción debe resolver, frente a diversas posibilidades, el cómo se articulan las cosas reales con «la» realidad. La realidad impele a realizarse, pero no cómo. ¿Cómo se realiza el hombre en sentido radical?

Veíamos que, desde el punto de vista «intelectivo», el hombre se ve impelido a buscar cuál es el «contenido fundamental» de la realidad para poder realizarse en sus acciones. Por ejemplo, no se vive de la misma manera si se entiende que el fundamento último de las acciones («la» realidad) es una realidad personal (Dios), la facticidad del ser (ateísmo), la propia vida (indiferencia), etc. Esta intelección se realiza con todos sus momentos (aprehensión primordial, logos y razón). Pero no basta con la intelección. La fundamentalidad, como veíamos, impele a tomar una opción o entrega (momento volitivo). Esta entrega no depende proporcionalmente de la firmeza o seguridad con que se intelija algo. Es posible, por ejemplo, una gran entrega con una mínima intelección fundamental, y viceversa. Por ello, veíamos que Zubiri entiende las actitudes radicales ante la fundamentalidad desde un momento intelectivo y volitivo.

Pero este análisis, con toda su riqueza intelectiva y volitiva, no es suficiente. Ante todo, porque el hombre para realizarse no le bastan con las tendencias

$19 \quad$ HD, p. 89. 
naturales que posee, sino que tiene que esbozar un «sistema de posibilidades» para poder optar (volición) por cuál forma de realidad se va a dar. Así Zubiri nos lo dice: «La realidad es impelente. Impele, velis nolis, a esbozar un sistema de posibilidades entre las que el hombre tiene que optar y que constituyen la última instancia de su propia realidad $»^{20}$. Ahora bien, esta creación o «esbozo de sistema de posibilidades» puede realizarse de dos modos. Por un lado, se pueden crear posibilidades para «actualizar» la realidad. Son posibilidades descriptivas y explicativas de la realidad. Es una creación que depende predominantemente de su inteligencia. Como estas posibilidades son algo creado y no dado por naturaleza, aquí predomina el momento de logos y de razón. Es lo que Zubiri ha desarrollado en su obra Inteligencia sentiente. Pero, por otro lado, el hombre necesita crear posibilidades no sólo para actualizar, sino para «realizarse» en cada acción. Y aquí es donde creemos que interviene "con mayor fuerza» el momento de sentimiento. No es que el sentimiento no tenga ningún papel en el primer modo, pero es en el segundo donde adquiere su mayor vigor. Zubiri no ha explicitado el papel que juega el sentimiento en la creación de posibilidades, pero sí lo ha hecho uno de sus discípulos, Diego Gracia. Ocuparemos algunas de sus ideas para intentar completar este punto ${ }^{21}$.

Diego Gracia ha propuesto que así como en la inteligencia hay tres momentos (aprehensión primordial, logos y razón), del mismo modo existirían estos momentos, mutatis mutandi, en el sentimiento y la volición. En el caso del sentimiento, su correlato de aprehensión primordial sería el pulchrum. Y, en este sentido, habría que decir que el sentimiento de fundamentalidad tiene un momento de pulchrum, de atemperamiento a lo real en tanto real. Sería el momento radical de todo sentimiento: «Yo puedo tener la fruición y la complacencia en algo real no por las cualidades que tiene, sino pura y simplemente porque es real $\aleph^{22}$. La fundamentalidad nos atempera, y es en la aprehensión primordial donde acontece este sentimiento de complacencia radical, la fruición de «la» realidad que me fundamenta.

Pero este análisis no es suficiente. El hombre en esta fundamentalidad se ve impelido a realizarse. Por ello, para poder tomar opción, necesita de valores. No le basta con un mero sistema de posibilidades puramente conceptual. Se puede entender, por ejemplo, qué es la solidaridad, pero eso no implica que sea un valor para el que la entiende. En el valor entra, además, el momento de sentimiento. Lo que es valioso para nosotros toca nuestro tono emocional. Para quien lo sienta como valioso queda atemperado frente a ella.

En el caso de la realización humana, el sentimiento, a partir del pulchrum como sentimiento de fundamentalidad radical, jugaría el papel del «logos» creando valores (la estimativa) y emitiendo juicios de valor ${ }^{23}$. Por su parte, la

20 HD, p. 90.

21 Cf. Gracia, D., (2017). El poder de lo real. op. cit., pp. 492-516.

22 SSV, p. 345.

23 «La estimación juega un papel similar a la simple aprehensión, bien que por vía emocional, y que la valoración puede identificarse con el juicio, que en este caso se convierte en 
voluntad realiza estos valores. Ahora bien, como decíamos, para su «realización» el hombre debe esbozar un sistema de posibilidades de acción. Y lo que hace posible una acción no es sólo su momento inteligible, sino lo valioso que sea para nosotros. Y en el tema del valor, siguiendo a Diego Gracia, el sentimiento juega un papel crucial. El sentimiento permite diferenciar una posibilidad sobre otras. Los valores le dan «sentido» a la vida, permiten direccionar nuestras acciones. De aquí entonces que podemos postular que el sentimiento tiene un papel crucial en la dirección de búsqueda para la creación de un sistema de posibilidades de realización.

Un ejemplo de estos dos modos de creación y realización de posibilidades nos lo da el propio Zubiri, hablando de la relatividad de Einstein. La postulación y probación de la teoría de la relatividad, como teoría científica, depende, en gran medida, del momento intelectivo (logos y razón). Pero la teoría como «realización» personal de Einstein se halla direccionada por la belleza (sentimiento) que éste encuentra en tal teoría. Así nos dice:

En una de las múltiples conversaciones que tuvo la amabilidad de concederme, Einstein me decía un día, hablando precisamente de la Escuela de Viena, de Ernst Mach, que tanto invocaba su nombre: «Se ha dicho mucho que yo me he inspirado en la filosofía de Mach. No es verdad: yo no me he inspirado en el principio de la economía del pensamiento, me he inspirado en algo distinto: en que creo que la arquitectura de las leyes de la Naturaleza tiene que tener una belleza y una armonía. Y la Relatividad se la da». Evidentemente. Pero esto no fue el enunciado del principio de relatividad. Fue la fruición estética que este hombre tenía y que dirigió su descubrimiento de la relatividad, que es cosa diferente ${ }^{24}$.

La veracidad del principio de relatividad, por ejemplo, no depende predominantemente de la fruición del científico; su belleza o atemperamiento a ella no decide totalmente su verdad, pero sí es lo que «dirige» su descubrimiento, lo que mueve a las diversas «acciones» que tiene que hacer Einstein para postular y demostrar esta teoría.

Ahora bien, tenemos que meditar todo esto en el sentimiento de una realidad-fundamento. Es el sentimiento de fundamentalidad en función de la «realización» de posibilidades de la forma de ser radical del hombre. Al respecto, recordemos que Zubiri llamaba a las actitudes ante la fundamentalidad (teísmo, ateísmo, etc.) «direcciones de búsqueda». No son un mero «producto» de un proceso racional ${ }^{25}$, sino que «dirigen» la búsqueda de los esbozos ante la fundamentalidad para realizarse. La intelección, decíamos, esboza el

juicio de valoración» (GRAcIa, D., (2017). El poder de lo real. op. cit., p. 511).

24 SSV, p. 349.

25 En la primera edición de HD, se colocó como segunda parte lo que en la nueva versión se llamó «El despliegue del problema de Dios». En este texto el tema de las actitudes se trata al final de la segunda parte luego del despliegue del proceso racional. Pero tanto en el «Curso de Roma» de HD (1973) como en lo que nos quedó redactado de la segunda parte (1983) el tema de las actitudes inicia la segunda parte. Esto parece manifestar que Zubiri 
contenido fundamental y la volición la entrega. Pero el sentimiento dirige la búsqueda. ¿Cómo? Sería través de los valores. Busca "posibilidades» de realización. Los valores dan «sentido» a la realización personal. Sería el papel de la estimativa radical: crear valores que permitan «dirigir» los diversos esbozos de las actitudes radicales.

En definitiva, en la aprehensión primordial se nos actualiza la realidad uno de cuyos momentos, desde el sentimiento afectante, es el ámbito del pulchrum. Según Gracia, desde allí, el hombre construye libremente, por vía de la estimación, diversos valores. Es el logos emocional o del sentimiento. En el caso del problema de la fundamentalidad, serán las diversas actitudes las que darán origen a los esbozos que el hombre deberá «probar» como solución al problema de la fundamentalidad.

\section{b) El carácter probatorio del sentimiento de fundamentalidad}

Proponíamos que el sentimiento de fundamentalidad dirige la búsqueda de posibles esbozos ante el problema de la fundamentalidad como el teísmo, ateísmo, indiferencia, etc. Es algo que en rigor se crea desde la intelección, sentimiento y volición, pero a nivel del logos. En este nivel no se trata de la creación propiamente tal de estos esbozos sino de aquello que permite saber dónde buscarlos. Son las «actitudes» radicales. Pero también proponemos que el sentimiento tendría un papel crucial en la "probación» de esos esbozos en el plano de la razón. No sólo orientarían su creación, sino también jugarían un papel importante en su probación y verificación.

Para explicar esto, pongamos primero atención al momento «intelectivo» donde se crean y prueban los esbozos. La razón crea el esbozo, el contenido fundamental de lo que sería la realidad-fundamento. Para el caso del problema de la fundamentalidad, la razón puede postular, por ejemplo, un teísmo donde la realidad-fundamento se identifica con un Dios personal, creador, omnipresente, forjador de valores radicales, etc. Pero puede postularse también que la realidad-fundamento se identifica con la facticidad del ser, con la propia vida, etc. Pero no basta con el esbozo, hay que probarlo, verificarlo. Es lo que Zubiri llama experiencia. Esta verificación es un proceso racional que no termina nunca. Es un «estar en vía». Por ello, nos dice que «verificar es siempre y sólo ir verificando. $\mathrm{Y}$ este ir verificando es lo que constituye la experiencia» ${ }^{26}$.

Hay que destacar aquí dos aspectos de esta verificación. Por una parte, hay tres modos básicos de verificación del esbozo: por sus «consecuencias» confirmables (si la hipótesis es cierta, entonces deben darse tales y cuales consecuencias), por su «concordancia» y por su «convergencia». Por otra parte, hay que atender a qué tipo de verificación se da. La verificación puede ser "adecuada» (lo cual se da pocas veces), «parcial» (lo real se hace razonable o viable) o

entendió las actitudes no sólo desde la razón, sino también desde el logos como «direcciones de búsqueda».

26 IRA, p. 269. 
«inverificable» (que es un tipo de verificación), donde hay, a su vez, dos tipos, lo refutable (experiencia negativa) y lo suspensivo.

Desde aquí habría que meditar la experiencia de la razón fundamental en sus múltiples vías (ateísmo, agnósticas, ateas, etc.). Por ejemplo, meditar cómo se ha dado la verificación del teísmo en el propio Zubiri. La verificación del esbozo teísta, por ejemplo, sería «parcial», nunca adecuada. Por otra parte, por su tipo de verificación, no parecería ser exactamente por sus «consecuencias confirmables», por ejemplo, someter «a prueba» a los dioses, algo así como decir que si Dios (o los dioses) existen, entonces deberían darse tales y cuales consecuencias ante un petitorio, etc. Como Antonio González ha sostenido, las religiones han tratado de huir de un "principio retributivo», es decir, del vivir del fruto de las acciones ${ }^{27}$. Las divinidades no se verifican de esta manera, en cierta forma son infalsables en este sentido consecuencialista. Para Zubiri, más bien podrían verificarse por «convergencia» cuyo punto último se daría, tal vez, en la otra vida. Así nos lo dice:

El sentido de la verdad religiosa no es precisamente el de una conformidad ni el de una adecuación. Es un sentido distinto: es el sentido de una vía. El que está convencido de la verdad de su fe evidentemente cree que, si se prolongara esa línea y se llegara a estar cara a cara al Dios en quien tiene fe, este Dios podría ser muy distinto de lo que se ha imaginado, pero desde luego corroboraría el camino que ha emprendido para llegar a Él. Esto es lo único que puede pretender la verdad religiosa. Una verdad religiosa que consiste en este "hacia» en que el hombre dirige su pensamiento hacia una divinidad concebida en la línea de una supremacía por un pensar religioso ${ }^{28}$.

El ateísmo, por su parte, como tipo de verificación, puede negar la verificación de estos esbozos teístas. Es la experiencia de inverificabilidad por refutación. También puede ocurrir que se dé una experiencia de suspensión del esbozo teísta: no se verifica ni se refuta. Sería el caso de algún tipo de agnosticismo.

Pero toda esta experiencia del esbozo fundamental afecta sólo al momento «intelectivo». Es lo que en general importó en esa disciplina filosófica llamada «teología natural» o «teodicea». Pero aquí se trata de una experiencia integral del hombre entero, una experiencia de realización personal. Por ello, hay que completarla con el sentimiento y la volición. Ya decíamos que la volición radical es la «entrega» ante la fundamentalidad esbozada. Y ésta, decíamos, no es proporcional a lo racional. Puede haber un gran conocimiento, y poca entrega, o al revés. El otro momento es el sentimiento. Y, a nuestro juicio, este momento es clave para la «probación» de los esbozos fundamentales. El hombre puede

27 Cfr., González, A., (1999). Teología de la praxis evangélica. Santander: Editorial Sal Terrae, pp. 134-156. Aquí Antonio González caracteriza a la historia de las religiones como un proceso de liberación del esquema de la ley, como liberación de la retribución que ella implica.

${ }_{28}$ ZuBIRI, X. (2015). El problema teologal del hombre: Dios, religión, cristianismo (PTH). Madrid: Alianza, p. 254. 
sentirse atemperado o no a lo que la razón propone como esbozo. Frente al esbozo puede hallarse cómodo o incómodo en su propia vida. Para ello, más que explicarlo, vamos a dar algunos testimonios tomados de la vida de algunos filósofos.

El primero procede del propio Zubiri. Cuando Zubiri era joven sintió una crisis al profesar cierto agnosticismo.

[...] veo que mi espíritu cae fatigado y exánime dejándose llevar por las oleadas de un agnosticismo irremediable [...] El sentimiento me dice: «Si no crees, tu vida carece de sentido, nada podrá proporcionarte la satisfacción de vivir, pues has roto con todo lo que educó tu espíritu y te provocó incomparables emociones». La razón protesta: «La vida afectiva puede ser una faena habitual por la fuerza de la educación; lo único importante es la meta de la verdad ${ }^{29}$.

Aquí puede apreciarse un conflicto entre lo que la razón verifica (un agnosticismo, una verificación «suspensiva») y lo que sus sentimientos le muestran: una vida «insatisfecha» (no atemperada), carente de sentido frente a ese esbozo suspensivo. ¿Será este sentimiento de «insatisfacción» el motor interno que provocó que Zubiri no se quedara en tal agnosticismo y le instara a buscar nuevos esbozos y experiencias de «Dios»?

Pero también podemos encontrar ciertos testimonios «ateos» que niegan cierto tipo de esbozo de «Dios». El primero lo extraemos de Nietzsche en su Zaratustra: «Mas para revelaros totalmente mi corazón a vosotros, amigos: si hubiera dioses, ¡cómo soportaría yo el no ser Dios! Por tanto, no hay dioses» ${ }^{30}$. Y más abajo señala: «Lejos de Dios y de los dioses me ha atraído esa voluntad; ¡qué habría que crear si los dioses - existiesen!» ${ }^{31}$.

El asunto es entender aquí lo que significa «Dios». Al parecer, dicho zubirianamente, el esbozo «Dios» es el de un ser donador de valores que deja al hombre sin creación de ellos. Y esto, para Nietzsche, revelando su corazón, sus sentimientos, es algo «insoportable». En su vida no cabe la experiencia de un «Dios» de este tipo. No se trata de la negación de todo Dios, sino de un esbozo particular de Dios. ¿Será esto lo que quiere decir Nietzsche con la «muerte de Dios»? ¿No abre esta muerte un espacio para nuevos esbozos?

Finalmente, recogemos el testimonio autobiográfico de Sartre.

Sólo una vez tuve el sentimiento de que existía [Dios]. Había jugado con unos fósforos y quemado una alfombrita. Estaba tratando de arreglar mi destrozo cuando, de pronto, Dios me vio, sentí Su mirada en el interior de mi cabeza y en las manos; estuve dando vueltas por el cuarto de baño, horriblemente visible, como un blanco vivo. Me salvó la indignación; me puse furioso contra tan grosera indiscreción, blasfemé, murmuré como mi abuelo: «Maldito Dios, maldito Dios, maldito Dios». No me volvió a mirar nunca más.

29 Carta de X. Zubiri a Benigno Pérez 18-4-1921, citado de Corominas, J. y Vicens, J. (2006). Xavier Zubiri. La soledad sonora. Madrid: Taurus, p. 112.

30 Nietzsche, F., (1987). Así habló Zaratustra. Madrid: Alianza, p. 132.

31 Ibid., p, 133. 
Acabo de contar la historia de una vocación fallida: yo necesitaba a Dios, me lo dieron, pero lo recibí sin comprender que lo buscaba. Al no poder enraizar en mi corazón, vegetó en mí durante algún tiempo y después se murió. Hoy, cuando me hablan de Él, digo con la diversión sin pena de un viejo que se encuentra con una vieja amiga: «Hace cincuenta años, sin ese malentendido, sin esa equivocación, sin el accidente que nos separó, podría haber habido algo entre nosotros» ${ }^{32}$.

Aquí nuevamente hay que tratar de entender lo que significa el esbozo «Dios» en Sartre. Se trata, al parecer, de un ser «omnipresente» y, de algún modo, sancionador. En la experiencia de quemar la alfombrita, Sartre se «indigna» ante la omnipresencia divina. Es una «indiscreción» insoportable. Lo que para algún creyente la omnipresencia da seguridad, para Sartre hace la vida imposible. Por ello, «Dios» no volvió a aparecer de esa forma. Tal sentimiento, ciertamente no decide todo lo que filosóficamente ha desarrollado después Sartre sobre «Dios», pero sí indica que es un momento esencial de su experiencia fundamental. Lo interesante es que, cuando lo mira en retrospectiva, con más edad, él mismo descubre que ha habido un "malentendido», una "equivocación». ¿Significa esto de que Sartre mismo entendió que pudo haber tenido un mejor esbozo de lo que es «Dios»? Y, por ello, ¿reconoce entonces que podría haber tenido una experiencia fundamental distinta en su vida?

Con estos testimonios intentamos mostrar qué papel podría tener el sentimiento de fundamentalidad en Zubiri en su momento de razón.

Pontificia Universidad Católica de Valparaíso, Chile

EsTEBAN VARGAS

esteban.vargas@pucv.cl

eugenia.colomer@pucv.cl

Eugenia Colomer

[Artículo aprobado para publicación en enero de 2019]

32 SARTRE, J., (1982). Las palabras. Madrid: Alianza, p. 71. 